\title{
Synthesis and Cytotoxic Activity of Arenecarbaldehyde 2-Pyridinylhydrazone Derivatives
}

\author{
Alessandra Campbell Pinheiro, ${ }^{a}$ Sarah Sant'Anna Maranhão, ${ }^{b}$ Guilherme Graziany Camelo Carvalho, ${ }^{b}$ \\ Augusto César Aragão Oliveira, ${ }^{b}$ Cláudia Pessoa,${ }^{b}$ Thaís Cristina Mendonça Nogueira, ${ }^{a}$ \\ Cristiane França da Costa, ${ }^{a}$ and Marcus Vinícius Nora de Souza ${ }^{*, a, c}$ \\ ${ }^{a}$ Fundação Oswaldo Cruz, Instituto de Tecnologia em Fármacos-Far Manguinhos, 21041-250, Rio de Janeiro, \\ RJ, Brazil \\ ${ }^{b}$ Laboratório de Oncologia Experimental, Núcleo de Pesquisa e Desenvolvimento de Medicamentos, \\ Universidade Federal do Ceará, Rodolfo Teófilo, 60430-275, Fortaleza, CE, Brazil

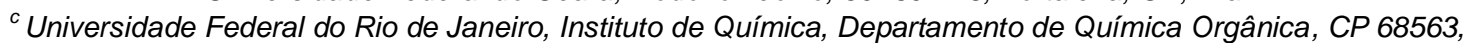 \\ 21945-970, Rio de Janeiro, RJ, Brazil \\ Email: marcus.nora@far.fiocruz.br (M. S.)
}

\begin{abstract}
Fourteen arenecarbaldehyde 2-pyridinylhydrazone derivatives have been synthesized and evaluated for their cytotoxic potential against four cancer cell lines using the MTT assay. The products were characterized by ${ }^{1} \mathrm{H}$ NMR, ${ }^{13} \mathrm{C}$ NMR, and HRMS, and the cytotoxicity results were expressed as the concentration that induced $50 \%$ inhibition of cell growth (IC50) in $\mu \mathrm{M}$. The most active compound was 2-pyridinecarbaldehyde 2-pyridinylhydrazone, with an $\mathrm{IC}_{50}$ value of $0.96 \mu \mathrm{M}$, which displayed potent and selective activity against the human prostate cancer cell line, PC3, similar to the antineoplastic drug doxorubicin.
\end{abstract}

Keywords cancer, hydrazone, 2-pyridinylhydrazones, PC3 cancer cell line, MTT assay

\section{Introduction}

Nitrogen heteroarene structural units are present in many marketed drugs. Currently, they represent $59 \%$ of all U.S. FDA approved pharmaceuticals. ${ }^{[1]}$ Pyridine is the most commonly used nitrogen heteroarene nucleus presenting in several anti-cancer drugs, for example, crizotinib and abiraterone (Figure 1), ${ }^{[2]}$ two novel chemotherapeutic drugs approved by FDA to treat non-small-cell lung cancer and metastatic prostate cancer, respectively. Nowadays, various pyridine derivatives are being developed and assessed in clinical trials for different types of cancer ${ }^{[3]}$ as well as in other biological areas. ${ }^{[4-6]}$ The examples of pyridine compounds being studied include metal complexes, ${ }^{[7,8]}$ imidazolyl-pyridine ${ }^{[9,10]}$ and pyrazolyl-pyridine derivatives. ${ }^{[11]}$<smiles>CC(=O)O[C@H]1CCC2C(=CC[C@@H]3C2CCC2(C)C(c4cccnc4)=CCC32)C1</smiles>

Abiraterone

Figure 1 Pyridine nucleus in anticancer drugs: Crizotinib (lung cancer) and Abiraterone (prostate cancer).

The great majority of antitumor drugs are usually related to an elevated number of non-specific toxicities, thus we urgently need more efficient and selective chemotherapeutics compounds to treat cancer. ${ }^{[12]}$

The hydrazonyl functional group, $\mathrm{CH}=\mathrm{N}-\mathrm{NH}$, readily formed from hydrazines and arenecarbaldehydes, is found in many compounds with a wide range of pharmacological activities, including antibacterial, antifungal, anticonvulsant, anti-inflammatory, antimalarial, antitubercular, and anti-cancer activities. ${ }^{[13-22]}$ In our earlier studies on anticancer activities of heteroaromatic compounds, we identified a series of hydrazonyl compounds with promising antitumoral activities based on the isonicotinoyl, ${ }^{[23]}$ quinolinyl, ${ }^{[24]}$ and quinoxalinyl ${ }^{[25-27]}$ frameworks. In our continuing search for new potent and safe antineoplastic agents and on the basis of the results of these previous studies, we have investigated the potential of pyridin2-yl-hydrazones, arene- $\mathrm{CH}=\mathrm{N}-\mathrm{NH}$-pyridin-2-yl derivatives as anticancer agents. Herein, we wish to report our findings.

\section{Results and Discussion}

\section{Synthesis and characterization}

The synthesis of the pyridin-2-yl-hydrazone derivatives, I, involved reactions of commercial pyridin-2-yl-hydrazine, II, and arenecarbaldehydes in ethanol solution at room temperature (Scheme 1 and Table 1). The selection of aldehydes used in the current study was based on those found in our earlier studies to produce strong cytotoxic activities in arenecarbaldehyde hydrazones. ${ }^{[28]}$ The compounds were obtained in $37 \%-89 \%$ yields (Table 1).

Scheme 1 Synthesis of 2-pyridin-2-yl-hydrazone derivatives la-1n

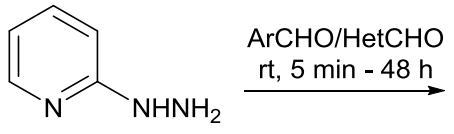

II<smiles>[Al]C=NNc1ccccn1</smiles>

la -1 n, $R=A r, 37 \%-89 \%$ 
Table 1 Pyridin-2-yl-hydrazone derivatives la-1n

\begin{tabular}{|c|c|c|c|c|}
\hline $\mathrm{R}$ & m.p. $/{ }^{\circ} \mathrm{C}$ & Yield/\% & $\operatorname{cog} P^{a}$ & $\begin{array}{l}\text { Mutagenic/ } \\
\text { Tumorigenic }^{b}\end{array}$ \\
\hline la $2-\mathrm{HOC}_{6} \mathrm{H}_{4}$ & $215-216$ & 65 & 2.27 & None/None \\
\hline lb 2,3-(HO) ${ }_{2} \mathrm{C}_{6} \mathrm{H}_{3}$ & $193-195$ & 65 & 1.57 & High/None \\
\hline Ic $2,4-(\mathrm{HO})_{2} \mathrm{C}_{6} \mathrm{H}_{3}$ & $220-221$ & 81 & 1.77 & None/None \\
\hline Id $2,5-(\mathrm{HO})_{2} \mathrm{C}_{6} \mathrm{H}_{3}$ & $230-231$ & 42 & 1.77 & None/None \\
\hline le $2-\mathrm{HO}-4-\mathrm{MeC}_{6} \mathrm{H}_{3}$ & $220-222$ & 64 & 2.70 & None/None \\
\hline If $2-\mathrm{HO}-3-\mathrm{MeOC}_{6} \mathrm{H}_{3}$ & $160-161$ & 72 & 1.88 & High/None \\
\hline Ig 2-HO-4- $\mathrm{MeOC}_{6} \mathrm{H}_{3}$ & $159-161$ & 44 & 2.30 & None/None \\
\hline Ih 2,6-(MeO) $)_{2} \mathrm{C}_{6} \mathrm{H}_{3}$ & $185-187$ & 89 & 2.35 & None/None \\
\hline li $3-\mathrm{O}_{2} \mathrm{~N}-4-\mathrm{Cl} \mathrm{C}{ }_{6} \mathrm{H}_{3}$ & $275-276$ & 71 & 2.90 & None/None \\
\hline lj $2-\mathrm{C}_{5} \mathrm{H}_{4} \mathrm{~N}$ & $178-179$ & 52 & 1.16 & Low/None \\
\hline Ik $3-\mathrm{C}_{5} \mathrm{H}_{4} \mathrm{~N}$ & $180-182$ & 70 & 1.09 & None/None \\
\hline II $4-\mathrm{C}_{5} \mathrm{H}_{4} \mathrm{~N}$ & $201-202$ & 74 & 1.04 & None/None \\
\hline Im 5- $\mathrm{O}_{2} \mathrm{~N}$-Furan-2-yl & $199-201$ & 37 & 1.67 & High/High \\
\hline In $5-\mathrm{O}_{2} \mathrm{~N}$-Thien-2-yl & $260-161$ & 78 & 2.31 & None/None \\
\hline
\end{tabular}

${ }^{a}$ Calculated using online www.molinspiration.com website.

${ }^{b}$ Calculated using Data Warrior program.

All compounds were identified from ${ }^{1} \mathrm{H} N M R,{ }^{13} \mathrm{C}$ NMR, IR spectral, and mass spectrometric data. In general, the ${ }^{1} \mathrm{H}$ NMR spectra showed the pyridine protons and the $\mathrm{N}=\mathrm{CH}$ proton in the ranges between $\delta 8.17-6.71$ and $7.97-8.32$, respectively. In the IR spectra, $v(\mathrm{~N}=\mathrm{C})$ was observed near to $1585 \mathrm{~cm}^{-1}$ in all cases.

\section{Cytotoxicity against cancer cell lines}

Initially, all 14 compounds were tested in vitro against three human cancer cells: HCT-116 (colon), SF-295 (glioblastoma), and HL-60 (leukemia) (National Cancer Institute, Bethesda, MD) using MTT assay $(5 \mu \mathrm{g} / \mathrm{mL}$ ) (Table 2). From these initial findings, compounds were classified into three activity groupings based on their growth inhibitions, GI, of at least one cell line: (a) active with $100 \%$ GI), (b) moderately active with 75 to $100 \%$ GI, and (c) poorly active to non-active with $\mathrm{Gl}>50 \%$.

Compounds included in groups $a$ and $b$ were found to be the salicylaldehyde derivatives, Ib, Ic, Id, le and If and the 2-pyridinyl derivative, $\mathbf{l j}$. These compounds were further investigated for in vitro cytotoxicity evaluation against four human cancer cell lines: HCT-116 (colon), PC3 (prostate), SF-295 (glioblastoma), and HL-60 (leukemia) using the MTT assay. Doxorubicin was used as a clinical reference drug, doxorubicin (Table 3).

The most active compound among the five tested in this second stage was 2-pyridinecarbaldehyde 2-pyridinylhydrazone $(\mathbf{I j})$, with a $50 \%$ inhibition of cell growth $\left(\mathrm{IC}_{50}\right)$, at 0.96 $\mu \mathrm{M}$, just a little greater than that $(0.76 \mu \mathrm{M})$ of the reference drug, doxorubicin, against the PC3 cell line. The structure-activity relationship (SAR) analysis indicated that the number, the positions, and the type of substituent to the aromatic ring are critical for the biological activity. It is considered significantly that in contrast to the activity of the 2-pyridinyl derivative $\mathbf{~ j}$, the 3- and 4-pyridinyl derivatives Ik and II were not active. Neither was the other heteroarene derivatives Im and In. Also, phenyl derivatives Ih and li was non-active. A property possessed by the salicylaldehyde derivatives and the 2-pyridinyl derivative $\mathbf{l j}$, is the ability to act as tridentate $\mathrm{O}, \mathrm{N}, \mathrm{N}$ - and $\mathrm{N}, \mathrm{N}, \mathrm{N}$-ligands, respectively, and thereby form strong complexes with metals. There are various reports in the literature of metal complexes formed from the salicylaldehyde and 2-pyridinyl ligands of type I. Typical examples are the complexes, chloro[(E)-pyridine-2carbaldehyde pyridine-2-ylhydronato]palladium(II), from the ligand $\mathbf{~ I j}{ }^{[29]}$ and metal complexes of $\mathbf{I}(\mathrm{Ar}=2-\mathrm{HO}-5-\mathrm{Br}-\mathrm{C} 6 \mathrm{H} 3) .{ }^{[30]}$
Table 2 Growth inhibition percentage (GI\%) for three tumors cells line by the MTT assay of compounds la-1n

\begin{tabular}{ccccccc}
\hline & \multicolumn{5}{c}{ Growth inhibition ${ }^{2} \%$} \\
\cline { 2 - 7 } I & HCT-116 & SD & SF-295 & SD & HL-60 & SD \\
\hline la & $81.10 \%$ & $0.28 \%$ & $73.13 \%$ & $4.32 \%$ & $45.49 \%$ & $46.55 \%$ \\
Ib & $80.05 \%$ & $0.65 \%$ & $78.71 \%$ & $2.42 \%$ & $96.75 \%$ & $0.00 \%$ \\
Ic & $86.50 \%$ & $2.70 \%$ & $73.23 \%$ & $0.56 \%$ & $84.91 \%$ & $0.37 \%$ \\
Id & $86.63 \%$ & $3.44 \%$ & $97.67 \%$ & $0.16 \%$ & $81.32 \%$ & $1.51 \%$ \\
le & $85.25 \%$ & $1.49 \%$ & $71.36 \%$ & $7.66 \%$ & $85.04 \%$ & $0.37 \%$ \\
If & $84.64 \%$ & $0.16 \%$ & $81.80 \%$ & $1.95 \%$ & $96.54 \%$ & $0.44 \%$ \\
Ig & $16.35 \%$ & $5.11 \%$ & $30.08 \%$ & $0.00 \%$ & $-30.49 \%$ & $11.04 \%$ \\
Ih & $74.32 \%$ & $0.81 \%$ & $68.26 \%$ & $1.95 \%$ & $93.50 \%$ & $1.04 \%$ \\
li & $27.42 \%$ & $0.65 \%$ & $30.15 \%$ & $1.02 \%$ & $6.23 \%$ & $7.85 \%$ \\
lj & $82.28 \%$ & $1.05 \%$ & $73.32 \%$ & $0.19 \%$ & $96.44 \%$ & $0.30 \%$ \\
Ik & $11.35 \%$ & $4.59 \%$ & $17.27 \%$ & $6.08 \%$ & $26.68 \%$ & $0.60 \%$ \\
II & $25.03 \%$ & $2.63 \%$ & $17.07 \%$ & $6.14 \%$ & $26.04 \%$ & $1.65 \%$ \\
Im & $2.82 \%$ & $5.11 \%$ & $14.90 \%$ & $4.55 \%$ & $-34.10 \%$ & $15.11 \%$ \\
In & $15.38 \%$ & $4.22 \%$ & $8.26 \%$ & $8,36 \%$ & $-1.94 \%$ & $2.96 \%$ \\
\hline
\end{tabular}

${ }^{a}$ Experiments were performed in triplicate. SD: Standard Deviation.

From the fact that this tridentate coordinating ability of the active compounds is a general one, it follows that a reasonable assumption for a possible mechanism is the removal of metal, vital for cell reproduction, on complexation by the drug.

Of some interest is the fact that compound la did not fall into the group of the more active compounds unlike the other salicylaldehyde derivatives, lb, Ic, Id, le, and If. Comparisons of the activities of $\mathbf{1 b}$ with that of $\mathbf{1 f}$, and that of Ic with those of le \& Ig, and also considering the poorer ability of la, point to the need of an additional strong coordinating group as well as the 2-hydroxy group within the salicylaldehyde unit for really good activity (Figure 2)

The toxicity of the compounds I was also determined using the Data Warrior program (Table 1). The most promising compounds Ic and lj were found to be non or low mutagenic/ tumorigenic.

Our research group also observed antileishmanial activities and antibacterial activities for these compounds, and our findings were previously reported. ${ }^{[1,32]}$

\section{Reported crystal structures of arenecarbaldehyde 2-pyridinylhydrazone derivatives}

The crystal structures of a number of $E$-arenecarbaldehyde 2-pyridinylhydrazone derivatives have been reported: these include Ic, Id, Ih, $\left[(\mathbf{I b}) \cdot 2 \mathrm{H}_{2} \mathrm{O}\right]$ and I (aryl $\left.=2-\mathrm{HO}-5-\mathrm{O}_{2} \mathrm{NC}_{6} \mathrm{H}_{3}\right) ;{ }^{[33]}$ lj; $;{ }^{[29]} \mathbf{I}\left(\mathrm{Ar}=4-\mathrm{XC}_{6} \mathrm{H}_{4}: \mathrm{X}=\mathrm{Br},{ }^{[34]} \mathrm{X}=4-\mathrm{Me}^{[35]}\right.$ and $\mathrm{X}=\mathrm{MeO} .^{[36]}$ The crystal structure determinations generally reveal that the molecules exhibit an $(E)$ arrangement at the hydrazonyl group and have nearly planar conformations, with angles between the aromatic rings in the main less than $5^{\circ}$ for anhydrous samples, but can be as large as $10^{\circ}$ in $\mathrm{Ih}^{\left[{ }^{[3]}\right.}$ Differences in the biological activities relate to factors other than differences in molecular conformations.

While the molecular conformations are similar, there are significant differences in the intermolecular interactions, especially with regards to the classical hydrogen bonds, 
Table 3 Cytotoxic activity of compounds $\mathbf{~ l b}$, Ic, Id, le, If and $\mathbf{l j}\left[\mathrm{IC}_{50}(\mu \mathrm{M})\right]$ on tumor cell lines ${ }^{a}$

\begin{tabular}{|c|c|c|c|c|}
\hline 1 & $\begin{array}{l}\text { HCT-116 } \\
\mathrm{IC}_{50} \\
\mathrm{Cl}\end{array}$ & $\begin{array}{l}\text { SF-295 } \\
\mathrm{IC}_{50} \\
\mathrm{Cl}\end{array}$ & $\begin{array}{l}\mathrm{HL}-60 \\
\mathrm{IC}_{50} \\
\mathrm{Cl}\end{array}$ & $\begin{array}{l}\mathrm{PC} 3 \\
\mathrm{IC}_{50} \\
\mathrm{Cl}\end{array}$ \\
\hline lb & $\begin{array}{l}7.34 \\
5.90 \text { to } 9.13\end{array}$ & $\begin{array}{l}8.60 \\
7.07 \text { to } 10.44\end{array}$ & $\begin{array}{l}0.96 \\
0.87 \text { to } 1.05\end{array}$ & $\begin{array}{l}9.47 \\
7.77 \text { to } 11.48\end{array}$ \\
\hline IC & $\begin{array}{l}3.14 \\
2.70 \text { to } 3.71\end{array}$ & $\begin{array}{l}4.76 \\
3.93 \text { to } 5.72\end{array}$ & $\begin{array}{l}0.19 \\
0.17 \text { to } 0.20\end{array}$ & $\begin{array}{l}5.46 \\
4.19 \text { to } 7.07\end{array}$ \\
\hline Id & $\begin{array}{l}9.04 \\
7.64 \text { to } 10.79\end{array}$ & $\begin{array}{l}18.60 \\
17.07 \text { to } 20.30\end{array}$ & $\begin{array}{l}1.05 \\
0.92 \text { to } 1.18\end{array}$ & $\begin{array}{l}5.41 \\
4.15 \text { to } 7.12\end{array}$ \\
\hline le & $\begin{array}{l}6.26 \\
4.93 \text { to } 7.93\end{array}$ & $\begin{array}{l}9.69 \\
8.37 \text { to } 11.23\end{array}$ & $\begin{array}{l}0.66 \\
0.48 \text { to } 0.88\end{array}$ & $\begin{array}{l}8.06 \\
6.04 \text { to } 10.75\end{array}$ \\
\hline If & $\begin{array}{l}6.09 \\
5.23 \text { to } 7.04\end{array}$ & $\begin{array}{l}5.60 \\
4.77 \text { to } 6.54\end{array}$ & $\begin{array}{l}1.44 \\
1.23 \text { to } 1.69\end{array}$ & $\begin{array}{l}6.95 \\
5.76 \text { to } 8.44\end{array}$ \\
\hline lj & $\begin{array}{l}1.01 \\
0.86 \text { to } 1.26\end{array}$ & $\begin{array}{l}4.14 \\
3.38 \text { to } 5.00\end{array}$ & $\begin{array}{l}0.23 \\
0.22 \text { to } 0.24\end{array}$ & $\begin{array}{l}0.96 \\
0.66 \text { to } 1.36\end{array}$ \\
\hline Doxo & $\begin{array}{l}0.21 \\
0.16-0.29\end{array}$ & $\begin{array}{l}0.41 \\
0.21-0.47\end{array}$ & $\begin{array}{l}0.02 \\
0.01-0.02\end{array}$ & $\begin{array}{l}0.76 \\
0.59-0.93\end{array}$ \\
\hline
\end{tabular}

${ }^{a}$ Data are presented as $\mathrm{IC}_{50}$ values and $95 \%$ confidence intervals $(\mathrm{Cl})$ obtained by nonlinear regression for all cell lines colon (HCT-116), glioblastoma (SF-295), leukemia (HL-60), and prostate (PC3), from three independent experiments. Doxorubicin was used as a positive control. Experiments were performed in triplicate. $\mathrm{IC}_{50}=$ concentrations that induce $50 \%$ inhibition of cell growth in $\mu \mathrm{M}$.

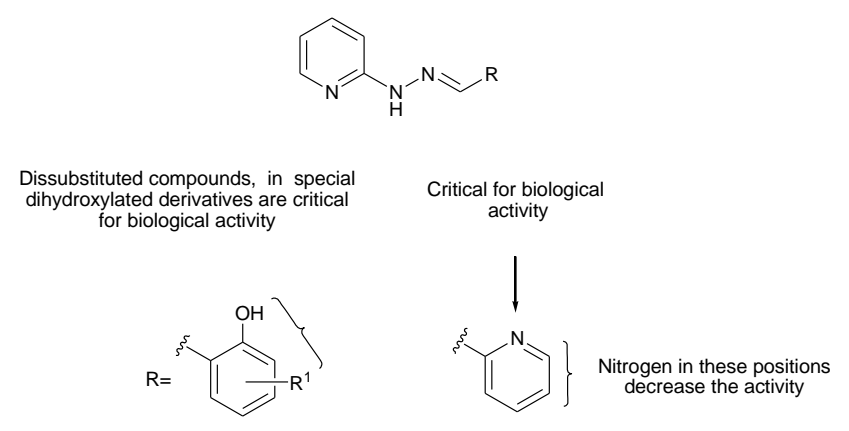

Figure 2 Important positions for cytotoxicity activity of these series against cancer cell lines.

$\mathrm{X}-\mathrm{H} \cdots \mathrm{Y}(\mathrm{X}$ or $\mathrm{Y}=\mathrm{O}$ or $\mathrm{N})$, exhibited by these compounds and which are dependent on the different substituents and patterns. Compounds, Id, le and I (2- $\mathrm{HO}-5-\mathrm{O}_{2} \mathrm{NC}_{6} \mathrm{H}_{3}{ }^{[33]}$ and I $\left(4-\mathrm{XC}_{6} \mathrm{H}_{4}\right.$ : $\mathrm{X}=\mathrm{Br},{ }^{[34]} \mathrm{X}=4-\mathrm{Me},{ }^{[35]}$ and $\mathrm{X}=\mathrm{MeO},{ }^{[36]}$ ) exhibit dimer forming N3-HN3 -..N1 (pyridinyl) hydrogen bonds: Id additionally has chain-forming $\mathrm{O} 2-\mathrm{H} 2 \cdots \mathrm{O} 1$ (hydroxyl) hydrogen bonds. In contrast, the classical intermolecular hydrogen bonds in Ic are chain-forming $\mathrm{N} 3-\mathrm{HN} 3 \cdots \mathrm{O} 1$ (hydroxyl) and $\mathrm{O} 2-\mathrm{H} 2 \cdots \mathrm{N} 1-$ (pyridinyl) hydrogen bonds. The hydrate molecule in $\left[(\mathrm{Ib}) \cdot 2\left(\mathrm{H}_{2} \mathrm{O}\right)\right]$ is very much involved in the hydrogen-bonding pattern, with $\mathrm{Ow}-\mathrm{Hw} \cdots \mathrm{N} 1$ intermolecular hydrogen bond occurring along with $\mathrm{N} 3-\mathrm{HN} 3 \cdots \mathrm{O} 2$ hydrogen bonds. Intramolecular $\mathrm{O} 1-\mathrm{H} 1 \ldots \mathrm{N} 2$ (hydrazonyl) hydrogen bonds are consistently found in each of the 2-hydroxyphenyl derivatives.

\section{Conclusions and Perspectives}

In this work, we report the cytotoxicity profile of a series of fourteen 2-pyridinyl hydrazone derivatives, which have been evaluated for their activity against human cancer cell lines. Compounds Ic and $\mathbf{l j}$ displayed the best cytotoxicity activities in all experiments. The compound 2-pyridinecarbaldehyde 2-pyridinylhydrazone (lj) showed potent and selective activity against the human prostate cancer cell line, PC3, with similar activity to that of doxorubicin. These results suggest that compounds Ic and lj could be useful starting points for further study for new anticancer drugs and confirm the potential of 2-pyridinylhydrazone derivatives as lead compounds in anticancer drug discovery.

\section{Material and Methods \\ Experimental}

Melting points were determined on Buchi apparatus. Infrared spectra were recorded on a Thermo Nicolet Nexus 670 spectrometer as potassium bromide pellets and frequencies are expressed in $\mathrm{cm}^{-1}$. Mass spectra (ESI assay in a solution of ammonium chloride) were recorded on Micromass ZQ Waters mass spectrometer. NMR spectra were recorded on a Bruker Avance 400 operating at $400.00 \mathrm{MHz}\left({ }^{1} \mathrm{H} \mathrm{NMR}\right)$ and $100.0 \mathrm{MHz}$ $\left({ }^{13} \mathrm{C} N M R\right)$ in DMSO- $d_{6}$. Chemical shifts are reported in ppm $(\delta)$ relative to tetramethylsilane and $J$-coupling in Hertz $(\mathrm{Hz})$. Proton and carbon spectra were typically obtained at room temperature.

\section{General procedure for synthesis of arenecarbaldehyde} 2-pyridinylhydrazone derivatives (I)

To a stirred solution of pyridin-2-yl-hydrazine $(1.0 \mathrm{mmol})$ in ethanol $(10 \mathrm{~mL})$ was added the appropriate benzaldehyde $(0.9$ $\mathrm{mmol}$ ), and the reaction mixture was stirred for $5 \mathrm{~min}-48 \mathrm{~h}$ at room temperature. The reaction mixture was concentrated under reduced pressure, and the residue was purified by washing with cold water $(3 \times 10 \mathrm{~mL})$, cold ether $(3 \times 10 \mathrm{~mL})$, or by column chromatographic purification using hexane/ethyl acetate $(50 \rightarrow 100 \%)$ as eluent, thus affording the pyridine-2-ylhydrazone derivatives I in 37\%-89\% yield.

(E)-2-((2-(Pyridin-2-yl)hydrazono)methyl)phenol (la): m.p. 215-216 ${ }^{\circ} \mathrm{C}$ (m.p. lit.: $216^{\circ} \mathrm{C}^{[37]}$ ); ${ }^{1} \mathrm{H}$ NMR (DMSO-d, $400 \mathrm{MHz}$ ) $\delta: 10.91(\mathrm{~s}, 1 \mathrm{H}), 10.51(\mathrm{~s}, 1 \mathrm{H}), 8.29(\mathrm{~s}, 1 \mathrm{H}), 8.13(\mathrm{dd}, J=4.9 \mathrm{~Hz}$ and $\left.J=1.9 \mathrm{~Hz}, 1 \mathrm{H}^{\prime}\right), 7.64$ (ddd, $\left.J=8.4,7.2,1.9 \mathrm{~Hz}, 1 \mathrm{H}\right), 7.58$ (dd, $J=7.6,1.6 \mathrm{~Hz}, 1 \mathrm{H}$ ), 7.18 (ddd, $J=8.3,7.6,1.6 \mathrm{~Hz}, 1 \mathrm{H}$ ), 7.03 (dd, $J=8.4,1.0 \mathrm{~Hz}, 1 \mathrm{H}$ ), 6.88 (dd, $J=8.3,1.0 \mathrm{~Hz}, 1 \mathrm{H}$ ), $6.87(\mathrm{dt}, J=7.6,1.0 \mathrm{~Hz}, 1 \mathrm{H}), 6.76$ (ddd, $J=7.2,4.9,1.0 \mathrm{~Hz}$, 
$1 \mathrm{H}) ;{ }^{13} \mathrm{C}$ NMR (DMSO-d6, $\left.100 \mathrm{MHz}\right) \delta: 156.3,155.8,147.8$, 138.5, 137.9, 129.6, 127.1, 120.4, 119.2, 115.9, 114.9, 106.0; IR (KBr) v: $1585 \mathrm{~cm}^{-1}$; HRESIMS m/z: $214.0980 \mathrm{C}_{12} \mathrm{H}_{12} \mathrm{~N}_{3} \mathrm{O}$ (calcd. 214.0975).

(E)-3-((2-(Pyridin-2-yl)hydrazono)methyl) benzene-1,2-diol (lb): m.p. $193-195{ }^{\circ} \mathrm{C}$ (m.p. lit.: $195-197{ }^{\circ} \mathrm{C}^{[38]}$ ); ${ }^{1} \mathrm{H}$ NMR (DMSO-d6, $400 \mathrm{MHz}$ ) $\delta: 10.92(\mathrm{~s}, 1 \mathrm{H}), 10.19$ (s, 1H), 9.24 (s, $1 \mathrm{H}$ ), $8.26(\mathrm{~s}, 1 \mathrm{H}), 8.14$ (dd, $J=4.8,1.4 \mathrm{~Hz}, 1 \mathrm{H}), 7.64$ (dt, $J=8.6$, $1.4 \mathrm{~Hz}, 1 \mathrm{H}), 6.99(\mathrm{~m}, 1 \mathrm{H}), 6.98(\mathrm{~d}, J=7.7 \mathrm{~Hz}, 1 \mathrm{H}), 6.78(\mathrm{~m}, 2 \mathrm{H})$, $6.69(\mathrm{t}, J=7.7 \mathrm{~Hz}, 1 \mathrm{H}) ;{ }^{13} \mathrm{C}$ NMR (DMSO- $\left.d_{6}, 100 \mathrm{MHz}\right) \delta: 156.2$, 148.0, 145.4, 144.6, 139.6, 138.0, 120.6, 119.1, 118.0, 115.8, 115.0, 106.1; IR (KBr) v: 3304, $1572 \mathrm{~cm}^{-1}$; HRESIMS $\mathrm{m} / \mathrm{z}$ : 230.0916 $\mathrm{C}_{12} \mathrm{H}_{12} \mathrm{~N}_{3} \mathrm{O}_{2}$ (calcd. 230.0924).

(E)-4-((2-(Pyridin-2-yl)hydrazono)methyl) benzene-1,3-diol (Ic): m.p. $220-221{ }^{\circ} \mathrm{C}$ (m.p. lit.: $220-221^{\circ} \mathrm{C}^{[39]}$ ); ${ }^{1} \mathrm{H}$ NMR (DMSO- $\left.d_{6}, 400 \mathrm{MHz}\right) \delta$ : $10.67(\mathrm{~s}, 2 \mathrm{H}), 9.71(\mathrm{~s}, 1 \mathrm{H}), 8.17(\mathrm{~s}, 1 \mathrm{H})$, $8.10(\mathrm{~d}, J=3.2 \mathrm{~Hz}, 1 \mathrm{H}), 7.61(\mathrm{~m}, 1 \mathrm{H}), 7.31(\mathrm{~d}, J=8.0 \mathrm{~Hz}, 1 \mathrm{H})$, $6.93(\mathrm{~d}, J=8.2 \mathrm{~Hz}, 1 \mathrm{H}), 6.72(\mathrm{~m}, 1 \mathrm{H}), 6.32(\mathrm{~m}, 1 \mathrm{H}), 6.31(\mathrm{~s}, 1 \mathrm{H})$; ${ }^{13} \mathrm{C}$ NMR (DMSO- $\left.d_{6}, 100 \mathrm{MHz}\right) \delta: 159.2,157.6,156.3,147.8$, 140.3, 137.8, 128.9, 114.4, 111.8, 107.4, 105.6, 102.4; IR (KBr) $v: 3238,1574 \mathrm{~cm}^{-1}$; HRESIMS $\mathrm{m} / z 230.0918 \mathrm{C}_{12} \mathrm{H}_{12} \mathrm{~N}_{3} \mathrm{O}_{2}$ (calcd. 230.0924).

(E)-2-((2-(Pyridin-2-yl)hydrazono)methyl) benzene-1,4-diol (Id): m.p. $230-231^{\circ} \mathrm{C} ;{ }^{1} \mathrm{H}$ NMR (DMSO- $\left.d_{6}, 400 \mathrm{MHz}\right) \delta$ : 10.83 (s, $1 \mathrm{H}), 9.68(\mathrm{~s}, 1 \mathrm{H}), 8.84(\mathrm{~s}, 1 \mathrm{H}), 8.21(\mathrm{~s}, 1 \mathrm{H}), 8.12(\mathrm{~m}, 1 \mathrm{H})$, $7.64(\mathrm{dt}, J=8.4,1.3 \mathrm{~Hz}, 1 \mathrm{H}), 7.03(\mathrm{~d}, J=8.4 \mathrm{~Hz}, 1 \mathrm{H}), 7.00(\mathrm{~d}$, $J=2.8 \mathrm{~Hz}, 1 \mathrm{H}), 6.76(\mathrm{~m}, 1 \mathrm{H}), 6.70(\mathrm{~d}, J=8.6 \mathrm{~Hz}, 1 \mathrm{H}), 6.62(\mathrm{dd}$, $J=8.6,2.8 \mathrm{~Hz}, 1 \mathrm{H}) ;{ }^{13} \mathrm{C}$ NMR (DMSO- $\left.d_{6}, 100 \mathrm{MHz}\right) \delta: 156.4$, 149.8, 148.6, 147.8, 138.2, 137.8, 120.7, 117.0, 116.6, 114.8, 111.9, 105.9; IR (KBr) v: 3351, $1585 \mathrm{~cm}^{-1}$; HRESIMS $\mathrm{m} / \mathrm{z}$ : 230.0919 $\mathrm{C}_{12} \mathrm{H}_{12} \mathrm{~N}_{3} \mathrm{O}_{2}$ (calcd. 230.0924).

(E)-5-Methyl-2-((2-(pyridin-2-yl)hydrazono) methyl)phenol (le): m.p. $220-222^{\circ} \mathrm{C}$ (m.p. lit.: $220^{\circ} \mathrm{C}^{[31]}$ ); ${ }^{1} \mathrm{H}$ NMR (DMSO- $d_{6}$, $400 \mathrm{MHz}) \delta$ : $10.84(\mathrm{~s}, 1 \mathrm{H}), 10.50(\mathrm{~s}, 1 \mathrm{H}), 8.24(\mathrm{~s}, 1 \mathrm{H}), 8.12(\mathrm{~m}$, $1 \mathrm{H}), 7.64$ (dt, $J=8.4,1.8 \mathrm{~Hz}, 1 \mathrm{H}), 7.43(\mathrm{~d}, J=7.7 \mathrm{~Hz}, 1 \mathrm{H}), 7.00$ $(\mathrm{d}, J=8.4 \mathrm{~Hz}, 1 \mathrm{H}), 6.76(\mathrm{~m}, 1 \mathrm{H}), 6.71(\mathrm{~s}, 1 \mathrm{H}), 6.69(\mathrm{~m}, 1 \mathrm{H})$, $2.26(\mathrm{~s}, 3 \mathrm{H}) ;{ }^{13} \mathrm{C}$ NMR (DMSO- $\left.d_{6}, 100 \mathrm{MHz}\right) \delta: 156.3,155.9$, 148.0, 139.7, 139.2, 138.0, 127.3, 120.3, 117.7, 116.4, 114.9, 106.0, 21.1; IR (KBr) v: $1584 \mathrm{~cm}^{-1}$; HRESIMS m/z: 228.1133 $\mathrm{C}_{13} \mathrm{H}_{14} \mathrm{~N}_{3} \mathrm{O}$ (calcd. 228.1131).

(E)-2-Methoxy-6-((2-(pyridin-2-yl)hydrazono) methyl)phenol (If): m.p. $160-161^{\circ} \mathrm{C}$ (m.p. lit.: $\left.160{ }^{\circ} \mathrm{C}^{[31]}\right) ;{ }^{1} \mathrm{H}$ NMR (DMSO- $d_{6}$, $400 \mathrm{MHz}) \delta: 10.91(\mathrm{~s}, 1 \mathrm{H}), 9.97(\mathrm{~s}, 1 \mathrm{H}), 8.3(\mathrm{~s}, 1 \mathrm{H}), 8.13(\mathrm{dd}$, $J=4.8,1.8 \mathrm{~Hz}, 1 \mathrm{H}), 7.64(\mathrm{dt}, J=8.6,1.8 \mathrm{~Hz}, 1 \mathrm{H}), 7.21(\mathrm{dd}$, $J=7.9,1.2 \mathrm{~Hz}, 1 \mathrm{H}), 7.06(\mathrm{~d}, J=8.6 \mathrm{~Hz}, 1 \mathrm{H}), 6.93(\mathrm{dd}, J=7.9$, $1.2 \mathrm{~Hz}, 1 \mathrm{H}), 6.80(\mathrm{t}, J=7.9 \mathrm{~Hz}, 1 \mathrm{H}), 6.77(\mathrm{~m}, 1 \mathrm{H}), 3.81(\mathrm{~s}, 3 \mathrm{H})$; ${ }^{1} \mathrm{H}$ NMR lit:: [40]; ${ }^{13} \mathrm{C}$ NMR (DMSO- $d_{6}, 100 \mathrm{MHz}$ ) $\delta: 156.4$, $147.8,147.8,145.3,138.2,137.9,120.8,118.9,118.6,114.9$, 112.0, 106.0, 55.7; IR (KBr) $v: 1573 \mathrm{~cm}^{-1}$; HRESIMS $\mathrm{m} / \mathrm{z}$ : 244.1072 $\mathrm{C}_{13} \mathrm{H}_{14} \mathrm{~N}_{3} \mathrm{O}_{2}$ (calcd. 244.1081).

(E)-5-Methoxy-2-((2-(pyridin-2-yl)hydrazono) methyl)phenol (Ig): m.p. $159-161^{\circ} \mathrm{C}$ (m.p. lit.: $160{ }^{\circ} \mathrm{C}^{[31]}$ ); ${ }^{1} \mathrm{H}$ NMR (DMSO- $d_{6}$, $400 \mathrm{MHz}) \delta: 10.81(\mathrm{~s}, 1 \mathrm{H}), 10.76(\mathrm{~s}, 1 \mathrm{H}), 8.21(\mathrm{~s}, 1 \mathrm{H}), 8.11(\mathrm{~d}$, $J=4.2 \mathrm{~Hz}, 1 \mathrm{H}), 7.63(\mathrm{~m}, 1 \mathrm{H}), 7.44(\mathrm{~d}, J=8.6 \mathrm{~Hz}, 1 \mathrm{H}), 6.96(\mathrm{~d}$, $J=8.4 \mathrm{~Hz}, 1 \mathrm{H}), 6.74(\mathrm{~m}, 1 \mathrm{H}), 6.48(\mathrm{~d}, J=8.6 \mathrm{~Hz}, 1 \mathrm{H}), 6.46(\mathrm{~s}$, 1H), 3.75 (s, 3H); ${ }^{1} \mathrm{H}$ NMR lit.: [41]; ${ }^{13} \mathrm{C}$ NMR (DMSO- $d_{6}, 100$ $\mathrm{MHz}) \delta: 160.8,157.6,156.3,148.0,139.8,138.0,128.8,114.7$, 113.4, 106.1, 105.8, 101.0, 55.2; IR (KBr) $v: 1570 \mathrm{~cm}^{-1}$; HRESIMS m/z: 244.1072 $\mathrm{C}_{13} \mathrm{H}_{14} \mathrm{~N}_{3} \mathrm{O}_{2}$ (calcd. 244.1081).

(E)-2-(2-(2,6-Dimethoxybenzylidene)hydrazinyl) pyridine (Ih): m.p. $185-187^{\circ} \mathrm{C}$ (m.p. lit.: $185{ }^{\circ} \mathrm{C}^{31}$ ); ${ }^{1} \mathrm{H}$ NMR (DMSO- $d_{6}$, $400 \mathrm{MHz}) \delta: 10.61(\mathrm{~s}, 1 \mathrm{H}), 8.22(\mathrm{~s}, 1 \mathrm{H}), 8.06(\mathrm{dd}, J=4.9,1.8$ $\mathrm{Hz}, 1 \mathrm{H}), 7.59$ (dt, $J=8.5,1.8 \mathrm{~Hz}, 1 \mathrm{H}), 7.26(\mathrm{t}, J=8.4 \mathrm{~Hz}, 1 \mathrm{H})$, $7.13(\mathrm{~d}, J=8.5 \mathrm{~Hz}, 1 \mathrm{H}), 6.71(\mathrm{~m}, 1 \mathrm{H}), 6.70(\mathrm{~d}, J=8.4 \mathrm{~Hz}, 2 \mathrm{H})$, $3.81(\mathrm{~s}, 6 \mathrm{H}) ;{ }^{13} \mathrm{C}$ NMR (DMSO- $\left.d_{6}, 100 \mathrm{MHz}\right) \delta: 158.1,158.1$, 157.3, 147.5, 137.6, 133.8, 129.5, 114.3, 112.0, 106.2, 104.4,
104.4, 55.9; IR (KBr) v: $1592 \mathrm{~cm}^{-1}$; HRESIMS m/z: 258.1232 $\mathrm{C}_{14} \mathrm{H}_{16} \mathrm{~N}_{3} \mathrm{O}_{2}$ (calcd.258.1237).

(E)-2-(2-(4-Chloro-3-nitrobenzylidene) hydrazinyl)pyridine (li): m.p. $275-276{ }^{\circ} \mathrm{C}$ (m.p. lit.: $275^{\circ} \mathrm{C}^{[31]}$ ); ${ }^{1} \mathrm{H}$ NMR (DMSO- $d_{6}$, $400 \mathrm{MHz}) \delta: 11.22(\mathrm{~s}, 1 \mathrm{H}), 8.31(\mathrm{~d}, J=1.7 \mathrm{~Hz}, 1 \mathrm{H}), 8.13$ (d, $J=4.1 \mathrm{~Hz}, 1 \mathrm{H}), 8.0(\mathrm{~s}, 1 \mathrm{H}), 7.93(\mathrm{dd}, J=8.4,1.7 \mathrm{~Hz}, 1 \mathrm{H}), 7.75$ $(\mathrm{d}, J=8.4 \mathrm{~Hz}, 1 \mathrm{H}), 7.66(\mathrm{~m}, 1 \mathrm{H}), 7.32(\mathrm{~d}, J=8.4 \mathrm{~Hz}, 1 \mathrm{H}), 6.81$ $(\mathrm{m}, 1 \mathrm{H}) ;{ }^{13} \mathrm{C}$ NMR (DMSO- $\left.d_{6}, 100 \mathrm{MHz}\right) \delta: 156.6,148.2,147.8$, 138.0, 136.2, 135.0, 131.8, 130.3, 123.5, 121.9, 115.7, 106.7; IR (KBr) v: 1581, 1525, $1348 \mathrm{~cm}^{-1}$; HRESIMS m/z: 277.0492 $\mathrm{C}_{12} \mathrm{H}_{10} \mathrm{ClN}_{4} \mathrm{O}_{2}$ (calcd. 277.0487).

(E)-2-((2-(Pyridin-2-yl)hydrazono)methyl) pyridine (lj): m.p. 178-179 ${ }^{\circ} \mathrm{C}$ (m.p. lit.: $179-180{ }^{\circ} \mathrm{C}^{[42]}$ ); ${ }^{1} \mathrm{H}$ NMR (DMSO- $d_{6}$, $400 \mathrm{MHz}) \delta: 11.16(\mathrm{~s}, 1 \mathrm{H}), 8.54(\mathrm{dd}, J=4.8,1.4 \mathrm{~Hz}, 1 \mathrm{H}), 8.14$ (dd, $J=4.9,1.0 \mathrm{~Hz}, 1 \mathrm{H}), 8.08(\mathrm{~s}, 1 \mathrm{H}), 7.96(\mathrm{~d}, J=8.0 \mathrm{~Hz}, 1 \mathrm{H})$, 7.80 (dt, $J=8.0,1.4 \mathrm{~Hz}, 1 \mathrm{H}$ ), 7.67 (ddd, $J=8.4,7.3,1.0 \mathrm{~Hz}$, $1 \mathrm{H}), 7.30$ (dd, $J=8.4,1.0 \mathrm{~Hz}, 1 \mathrm{H}), 7.28(\mathrm{~m}, 1 \mathrm{H}), 6.81$ (ddd, $J=$ 7.3, 4.9, $1.0 \mathrm{~Hz}, 1 \mathrm{H}$ ); ${ }^{13} \mathrm{C}$ NMR (DMSO- $\left.d_{6}, 100 \mathrm{MHz}\right) \delta: 156.6$, 154.2, 149.1, 147.7, 139.1, 137.9, 136.4, 122.8, 118.7, 115.5, 106.4; IR (KBr) v: $1571 \mathrm{~cm}^{-1}$; HRESIMS $\mathrm{m} / \mathrm{z}: 199.0986$ $\mathrm{C}_{11} \mathrm{H}_{11} \mathrm{~N}_{4}$ (calcd. 199.0978).

(E)-2-((2-(Pyridin-3-ylmethylene)hydrazinyl) pyridine (lk) m.p. $180-182{ }^{\circ} \mathrm{C}$ (m.p. lit.: $\left.179-180{ }^{\circ} \mathrm{C}^{[43]}\right) ;{ }^{1} \mathrm{H}$ NMR (DMSO- $\left.d_{6}, 400 \mathrm{MHz}\right) \delta: 11.07(\mathrm{~s}, 1 \mathrm{H}), 8.81(\mathrm{~d}, J=1.8 \mathrm{~Hz}, 1 \mathrm{H})$, 8.50 (dd, $J=4.7,1.5 \mathrm{~Hz}, 1 \mathrm{H}), 8.12(\mathrm{~m}, 1 \mathrm{H}), 8.08$ (ddd, $J=8.0$, 1.8, $1.5 \mathrm{~Hz}, 1 \mathrm{H}), 8.04(\mathrm{~s}, 1 \mathrm{H}), 7.65(\mathrm{dt}, J=8.4,1.8 \mathrm{~Hz}, 1 \mathrm{H})$, $7.41(\mathrm{dd}, J=8.0,4.7 \mathrm{~Hz}, 1 \mathrm{H}), 7.29(\mathrm{~d}, J=8.4 \mathrm{~Hz}, 1 \mathrm{H}), 6.79(\mathrm{~m}$, $1 \mathrm{H}) ;{ }^{13} \mathrm{C}$ NMR (DMSO- $\left.d_{6}, 100 \mathrm{MHz}\right) \delta: 156.8,149.0,147.7$, 147.5, 137.9, 135.4, 132.2, 131.2, 123.7, 115.2, 106.4; IR (KBr) $v$ : $1591 \mathrm{~cm}^{-1}$; HRESIMS m/z: $199.0984 \mathrm{C}_{11} \mathrm{H}_{11} \mathrm{~N}_{4}$ (calcd. 199.0978)

(E)-2-(2-(Pyridin-4-ylmethylene)hydrazinyl) pyridine (II): m.p. 201-202 ${ }^{\circ} \mathrm{C}$ (m.p. lit.: 202-204 ${ }^{\circ} \mathrm{C}^{[38]}$ ); ${ }^{1} \mathrm{H}$ NMR (DMSO-d $d_{6}$, $400 \mathrm{MHz}) \delta: 11.27(\mathrm{~s}, 1 \mathrm{H}), 8.56(\mathrm{~d}, J=5.9 \mathrm{~Hz}, 2 \mathrm{H}), 8.15(\mathrm{~m}, 1 \mathrm{H})$, $7.98(\mathrm{~s}, 1 \mathrm{H}), 7.69(\mathrm{dt}, J=8.5,1.7 \mathrm{~Hz}, 1 \mathrm{H}), 7.60(\mathrm{~d}, J=5.9 \mathrm{~Hz}$, $2 \mathrm{H}), 7.32(\mathrm{~d}, J=8.5 \mathrm{~Hz}, 1 \mathrm{H}), 6.84(\mathrm{~m}, 1 \mathrm{H}) ;{ }^{13} \mathrm{C}$ NMR (DMSO- $d_{6}$, $100 \mathrm{MHz}) \delta: 156.5,149.9,149.9,147.7,142.4,138.0,135.6$, $119.9,119.9,115.7,106.6$; IR $(\mathrm{KBr}) v: 1576 \mathrm{~cm}^{-1}$; HRESIMS $\mathrm{m} / \mathrm{z}: 199.0985 \mathrm{C}_{11} \mathrm{H}_{11} \mathrm{~N}_{4}$ (calcd. 199.0978).

(E)-2-(2-((5-Nitrofuran-2-yl)methylene) hydrazinyl)pyridine (Im): m.p. $199-201{ }^{\circ} \mathrm{C}$ (m.p. lit.: $\left.202{ }^{\circ} \mathrm{C}^{[44]}\right) ;{ }^{1} \mathrm{H}$ NMR (DMSO- $d_{6}$, $400 \mathrm{MHz}) \delta: 11.56(\mathrm{~s}, 1 \mathrm{H}), 8.17(\mathrm{~m}, 1 \mathrm{H}), 7.97(\mathrm{~s}, 1 \mathrm{H}), 7.79(\mathrm{~d}$, $J=4.0 \mathrm{~Hz}, 1 \mathrm{H}), 7.71$ (ddd, $J=8.4,7.4,1.8 \mathrm{~Hz}, 1 \mathrm{H}), 7.25(\mathrm{~d}, J=$ $8.4 \mathrm{~Hz}, 1 \mathrm{H}), 7.10(\mathrm{~d}, J=4.0 \mathrm{~Hz}, 1 \mathrm{H}), 6.88(\mathrm{~m}, 1 \mathrm{H}) ;{ }^{13} \mathrm{C}$ NMR (DMSO- $d_{6}, 100 \mathrm{MHz}$ ) $\delta: 155.9,153.5,151.1,147.8,138.2$, 126.3, 116.4, 115.5, 111.8, 106.8; IR (KBr) v: 1601, 1572, 1360 $\mathrm{cm}^{-1}$; HRESIMS m/z: $233.0663 \mathrm{C}_{10} \mathrm{H}_{9} \mathrm{~N}_{4} \mathrm{O}_{3}$ (calcd. 233.0669).

(E)-2-(2-((5-Nitrothiophen-2-yl)methylene) hydrazinyl)pyridine (In): m.p. $260-261^{\circ} \mathrm{C}$ (m.p. lit.: $\left.260{ }^{\circ} \mathrm{C}^{[45]}\right) ;{ }^{1} \mathrm{H}$ NMR (DMSO- $\left.d_{6}, 400 \mathrm{MHz}\right) \delta: 11.52(\mathrm{~s}, 1 \mathrm{H}), 8.17(\mathrm{~m}, 2 \mathrm{H}), 8.08(\mathrm{~d}, J=$ $4.4 \mathrm{~Hz}, 1 \mathrm{H}), 7.71(\mathrm{~m}, 1 \mathrm{H}), 7.35(\mathrm{~d}, J=4.4 \mathrm{~Hz}, 1 \mathrm{H}), 7.22(\mathrm{~d}, J=$ $8.4 \mathrm{~Hz}, 1 \mathrm{H}), 6.88(\mathrm{~m}, 1 \mathrm{H}) ;{ }^{13} \mathrm{C}$ NMR (DMSO- $\left.d_{6}, 100 \mathrm{MHz}\right) \delta$ : $155.9,148.9,148.7,147.8,138.2,131.6,130.9,126.3,116.3$, 106.7; IR (KBr) v: 1593, 1567, $1363 \mathrm{~cm}^{-1}$; HRESIMS $\mathrm{m} / \mathrm{z}$ 249.0436 $\mathrm{C}_{10} \mathrm{H}_{9} \mathrm{~N}_{4} \mathrm{O}_{2} \mathrm{~S}$ (calcd. 249.0441).

\section{Cytotoxicity against cancer cell lines}

Compounds I was tested for their cytotoxic activity against 3-4 human cancer cell lines: HCT-116 (colon), SF-295 (glioblastoma), HL-60 (leukemia), and PC3 (prostate) (National Cancer Institute, Bethesda, MD). Cell lines were maintained in RPMI 1640 medium supplemented with $10 \%$ fetal bovine serum, $2 \mathrm{mM}$ glutamine, $100 \mathrm{U} / \mathrm{mL}$ penicillin, and $100 \mu \mathrm{g} / \mathrm{mL}$ streptomycin at $37{ }^{\circ} \mathrm{C}$ with $5 \% \mathrm{CO}_{2}$. Each compound was dissolved in DMSO at a final concentration of $1 \mathrm{mg} / \mathrm{mL}$. After serial dilution, compounds were incubated with cell lines for 72 h. DMSO final concentration in the culture medium was kept 
constant and below $0.1 \%(V / V)$. The negative control received the same amount of DMSO $(0.001 \%$ in the highest concentration). After incubation, cell viability was determined by the reduction of the yellow dye 3-(4,5-dimethyl-2-thiazol)-2,5diphenyl-2H-tetrazolium bromide (MTT) to a blue formazan product as described by Mosmann. ${ }^{[46]}$

\section{Acknowledgment}

The authors are grateful to the Brazilian agency CNPq (Conselho Nacional de Desenvolvimento Cientifico e Tecnológico) for fellowships and financial support and the National Cancer Institute (Bethesda, MD, USA) for the donation of tumor 443 cell lines used in this study.

\section{Author Contributions}

Conceptualization, Marcos Nora and Claudia Pessoa; Formal analysis, Alessandra Campbell Pinheiro, Sarah Sant' Anna Maranhão, Guilherme Graziany Camelo Carvalho; Methodology, Alessandra Campbell Pinheiro, Sarah Sant' Anna Maranhão, Guilherme Graziany Camelo Carvalho; Project administration, Claudia Pessoa, Marco Nora; Writing, review \& editing, Augusto César Aragão Oliveira.

\section{Supporting Information}

Supporting information for this article is available on the WWW under www.genchemistry.org/EN/10.21127/yaoyigc202 00016.

\section{Conflict of interest}

The authors declare that there are no conflicts of interest.

Copyright @ 2020 Alessandra Campbell Pinheiro, Sarah Sant'Anna Maranhão, Guilherme Graziany Camelo Carvalho, Augusto César Aragão Oliveira, Cláudia Pessoa, Thaís Cristina Mendonça Nogueira, Cristiane França da Costa, and Marcus Vinícius Nora de Souza. This article is an open access article distributed under the terms and conditions of the Creative Commons Attribution (CC BY) license (http://creativecommons.org/licenses/by/4.0/). The use, distribution or reproduction in other forums is permitted, provided the original author(s) or licensor are credited and that the original publication in this journal is cited, in accordance with accepted academic practice. No use, distribution or reproduction is permitted which does not comply with these terms.

\section{References}

[1] Martins, P.; Jesus, J.; Santos, S.; Raposo, L. R.; Roma-Rodrigues, C.; Baptista, P. V.; Fernandes, A. R. Heterocyclic anticancer compounds: recent advances and the paradigm shift towards the use of nanomedicine's tool box. Molecules 2015, 20, 1685216891.

[2] Vitaku, E.; Smith, D. T.; Njardarson, J. T. Analysis of the structural diversity, substitution patterns, and frequency of nitrogen heterocycles among U.S. FDA approved pharmaceuticals. J. Med. Chem. 2014, 57, 10257-10274.

[3] Phrma Foundation. List of 2018 medicines in development for cancer. https://www.phrma.org/Report/List-of-2018-Medicines-inDevelopment-for-Cancer (Accessed July 18, 2020).

[4] Chaubey, A.; Pandeya, S. N. Pyridine: A versatile nucleus in pharmaceutical field. Asian J. Pharm. Clin. Res. 2011, 4, 5-8.

[5] Altaf, A. A.; Shahzad, A.; Gul, Z.; Rasool, N.; Badshah, A.; Lal, B.; Khan, E. A. A review on the medicinal importance of pyridine derivatives. J. Drug Des. Med. Chem. 2015, 1, 1-11.

[6] Ahmad, G.; Rasool, N.; Ikram, H. M.; Khan, S. G.; Mahmood, T.; Ayub, K.; Zubair, M.; Al-Zahrani, E.; Rana, U. A.; Akhtar, M. N.; Alitheen, N. B. Efficient synthesis of novel pyridine-based derivatives via suzuki cross-coupling reaction of commercially available 5-bromo-2-methylpyridin-3- amine: quantum mechanical investigations and biological activities. Molecules 2017, 22, 190.

[7] Gu, L.; Li, X.; Ran, Q.; Kang, C.; Lee, C.; Shen, J. Antimetastatic activity of novel ruthenium (III) pyridine complexes. Cancer Med. 2016, 5, 2850-2860.

[8] Fereidoonnezhad, M.; Niazi, M.; Ahmadipour, Z.; Mirzaee, T.; Faghih, Z.; Faghih, Z.; Shahsavari, H. R. Cyclometalated platinum(II) complexes comprising 2-(diphenylphosphino)pyridine and various thiolate ligands: synthesis, spectroscopic characterization, and biological activity. Eur. J. Inorg. Chem. 2017, 15, 2247-2254.

[9] Yu, Y.; Han, Y.; Zhang, F.; Gao, Z.; Zhu, T.; Dong, S.; Ma, M. Design, synthesis, and biological evaluation of imidazo[1,2-a]pyridine derivatives as novel PI3K/mTOR dual inhibitors. J. Med. Chem. 2020, 63, 3028-3046.

[10] Krause, M.; Foks, H.; Gobis, K. Pharmacological potential and synthetic approaches of imidazo[4,5-b]pyridine and imidazo[4,5-c]pyridine derivatives. Molecules 2017, 22, 399.

[11] Naik, N. S.; Shastri, L. A.; Shastri, S. L.; Chougala, B. M.; Shaikh, F.; Madar, J. M.; Kulkarni, R. C.; Dodamani, S.; Jalalpure, S.; Joshi, S. D.; Sunagar, V. Synthesis of polyfunctionalized fused pyrazolopyridines: characterization, anticancer activity, protein binding and molecular docking studies. ChemistrySelect 2019, 4, 285-297.

[12] WHO report on cancer: setting priorities, investing wisely, and providing care for all. https://apps.who.int/iris/handle/10665/ 330745 (Accessed July 18, 2020).

[13] Rollas, S.; Küçükgüzel, Ş. G. Biological activities of hydrazone derivatives. Molecules 2007, 12, 1910.

[14] Brum, J. O. C.; França, T. C. C.; LaPlante, S. R.; Villar, J. D. F. Synthesis and biological activity of hydrazones and derivatives: a review. Mini-Rev. Med. Chem. 2020, 20, 342-368.

[15] Świątek, P.; Saczko, J.; Rembiałkowska, N.; Kulbacka. Synthesis of new hydrazone derivatives and evaluation of their efficacy as proliferation inhibitors in human cancer cells. J. Med. Chem. 2019, 15, 903-910.

[16] Alotabi, S. H. Synthesis, characterization, anticancer activity, and molecular docking of some new sugar hydrazone and arylidene derivatives. Arab. J. Chem. 2020, 13, 4771-4784.

[17] Basiony, E. A.; Hassan, A. A.; Al-Amshany, Z. M.; Abd-Rabou, A. A.; Abdel-Rahman, A. A.; Hassan, N. A.; El-Sayed, W. A. Synthesis and cytotoxic activity of new thiazolopyrimidine sugar hydrazones and their derived acyclic nucleoside analogues. Molecules 2020, 25, 399.

[18] Nogueira, T. C. M.; Cruz, L. S.; Lourenço, M. C.; De Souza, M. V. N. Design, Synthesis and anti-tuberculosis activity of hydrazones and $\mathrm{N}$-acylhydrazones containing vitamin B6 and different heteroaromatic nucleus. Lett. Drug Des. Discov. 2019, 16, 792-798.

[19] Coimbra, E. S.; De Souza, M. V. N.; Terror, M.S.; Pinheiro, A. C.; Granato, J. T. Synthesis, biological activity, and mechanism of action of new 2-pyrimidinyl hydrazone and $\mathrm{N}$-acylhydrazone derivatives, a potent and new classes of antileishmanial agents. Eur. J. Med. Chem. 2019, 184, 111742.

[20] Dascalu, A.; Ghinet, A.; Lipka, E.; Furman, C.; Rigo, B.; Fayeulle, A.; Billamboz, M. Design, synthesis and evaluation of hydrazine and acyl hydrazone derivatives of 5-pyrrolidin-2-one as antifungal agents. Bioorg. Med. Chem. Lett. 2020, 30, 127220.

[21] Mathew, B.; Suresh, J.; Ahsan, M. J.; Mathew, G. E.; Usman, D.; Subramanyan, P. N. S.; Safna, K. F.; Maddela, S. Hydrazones as a privileged structural linker in antitubercular agents: a review. Infect. Disord. Drug Targets 2015, 15, 76-88.

[22] Popiołek, L. Hydrazide-hydrazones as potential antimicrobial agents: overview of the literature since 2010. Med. Chem. Res. 2017, 26, 287-301.

[23] Rodrigues, F. A. R.; Oliveira, A. C. A.; Cavalcanti, B. C.; Pessoa, C. O.; Pinheiro, A. C.; De Souza, M. V. N. Biological evaluation of 
isoniazid derivatives as an anticancer class. Sci. Pharm. 2014, 82, 21-28.

[24] Pinto, L. S. S.; De Souza, M. V. N.; Kaiser, C. R.; Wardell, J. L.; Wardell, S. M. S. V. N-(2-(Arylmethylimino)ethyl)-7-chloroquinolin4-amine derivatives: a new and potent class of anticancer agents. Lett. Drug Des. Discov. 2018, 15, 113-117.

[25] Rodrigues, F. A. R.; Bomfim, I. S.; Cavalcanti, B. C.; Pessoa, C. O.; Wardell, J. L.; Wardell, S. M. S. V.; Pinheiro, A. C.; Kaiser, C. R.; Nogueira, T. C. M.; Low, J. N.; Gomes, L. R.; De Souza, M. V. N. Design, synthesis and biological evaluation of (E)-2-(2-arylhydrazinyl)quinoxalines, a promising and potent new class of anticancer agents. Bioorg. Med. Chem. Lett. 2014, 24, 934-938.

[26] Maranhão, S. S.; Moura, A. F.; Oliveira, A. C. A.; Lima, D. J. B.; Barros-Nepomuceno, F. W. A.; Paier, C. R. K.; Pinheiro, A. C.; Nogueira, T. C. M.; De Souza, M. V. N.; Pessoa, C. Synthesis of PJOV56, a new quinoxalinyl-hydrazone derivative able to induce autophagy and apoptosis in colorectal cancer cells, and related compounds. Bioorg. Med. Chem. Lett. 2020, 30, 126851.

[27] Guerreiro, D. D.; De Lima, L. F.; De Sá, N. A. R.; Mbemya, G. T.; Ferreira, A. C. A.; Alves, B. G.; Maranhão, S. S.; Pessoa, C.; Pinheiro, A. C.; Nogueira, T. C. M.; De Souza, M. V. N.; De Figueiredo, J. R.; Rodrigues, A. P. R. Response of preantral follicles exposed to quinoxaline: A new compound with anticancer potential. Res. Vet. Sci. 2020, 128, 261-268.

[28] Rodrigues, F. A. R., Oliveira, A. C. A.; Cavalcanti, B. C.; Costa, P. M.; Pessoa, C. O.; Pinheiro, A. C.; De Souza, M. V. N. Cytotoxic Evaluation of Substituted Benzaldehydes. Eur. Chem. Bull. 2014, 3 555-558.

[29] Casey, A. T.; Hoskins, B. F.; Traverso, I. P. The crystal and molecular structures of the ligand $(E)$-Pyridine-2-carbaldehyde Pyridin-2'-ylhydrazone and the palladium(II) complex Chloro[(E)pyridine-2-carbaldehyde pyridin-2'-ylhydrazonato] palladium (II) which involves the coordination of the ligand in its anionic form. Aust. J. Chem. 1984, 37, 739-749.

[30] Shongwe, M. S.; Al-Barhi, K. S.; Mikuriya, M.; Adams, H.; Morris, M. J.; Bill, E.; Molloy, K. C. Tuning a single ligand system to stabilize multiple spin states of manganese: a first example of a hydrazone-based manganese(III) spin-crossover complex. Chem. Eur. J. 2014, 20, 9693-9701.

[31] Coimbra, E. S.; Antinarelli, L. M. R.; Crispi, M. A.; Nogueira, T. C. M.; Pinheiro, A. C.; De Souza, M. V. N. Synthesis, biological activity, and mechanism of action of 2-pyrazyl and pyridylhydrazone derivatives, new classes of antileishmanial agents. ChemMedChem 2018, 13, 1387-1394.

[32] Halicki, P. C. B.; Radin, V.; von Groll, A.; De Souza, M. V. N.; Pinheiro, A. C.; Da Silva, P. E. A.; Ramos, D. F. Antibiofilm potential of arenecarbaldehyde 2-pyridinylhydrazone derivatives against Acinetobacter baumannii. Microb. Drug Resist. 2019, in press. DOI: 10.1089/mdr.2019.0185.

[33] Correira, N. R. L.; Noguiera, T. C. M.; Pinheiro, A. C.; De Souza, M. V. N.; Wardell, J. L.; Wardell, S. M. S. V. Crystal structures of
(E)-2-((2-((pyridin-2-yl)hydrazonyl)methyl)phenolic compounds: different sets of classical hydrogen bonds, $\mathrm{X}-\mathrm{H} \cdots \mathrm{Y}(\mathrm{X}, \mathrm{Y}=\mathrm{O}, \mathrm{N}) . Z$. Kristallogr. 2016, 231, 271-284.

[34] Tunç, T.; Tezcan, H.; Şahin, E.; Dilek, N. Synthesis, crystal structure, and spectroscopic studies of $\mathrm{N}$-(4-bromobenzylidene)$N^{\prime}$-(2-pyridyl) hydrazine schiff base molecule. Mol. Cryst. Liq. Cryst. 2012, 552, 194-208.

[35] Yuvaraj, H.; Sundaramoorthy, S.; Velmurugan, D.; Kalkhambkar, R. G. (Z)-2-[2-(4-Methylbenzylidene)hydrazinyl]pyridine. Acta Crystallogr. 2011, E67, o178.

[36] Tunc, T.; Sari, M.; Yagbasan, R.; Tezcan, H.; Sahin, E. N-(4-Methoxybenzylidene)-N'-(2-pyridyl)hydrazine. Acta Crystallogr. 2003, C59, o192-0193.

[37] Syeda, N.; Srinivasan, V. R. Some fused s-triazoles derived from azaheterocyclic hydrazones. Indian J. Chem. 1965, 3, 162-167.

[38] Sandbhor, U.; Padhye, S.; Billington, D.; Rathbone, D.; Franzblau, S.; Anson, C. E.; Powell, A. K. Synthesis, x-ray crystal-structures, spectroscopic properties and antimycobacterial activity against Mycobacterium tuberculosis H37Rv. J. Inorg. Biochem. 2002, 90, 127-136.

[39] Cushman, M.; Nagarathnam, D.; Gopal, D.; Geahlen, R. L. Synthesis and evaluation of new protein-tyrosine kinase inhibitors. Part 2. Phenylhydrazones. Bioorg. Med. Chem. Lett. 1991, 1, 215-218.

[40] Patil, S. A.; Weng, C.; Huang, P.; Hong, F. Convenient and efficient Suzuki-Miyaura cross-coupling reactions catalyzed by palladium complexes containing N,N,O tridentate ligands. Tetrahedron 2009, 65, 2889-2897.

[41] Li, K.; Tong, A. A new fluorescent chemosensor for $\mathrm{Zn}^{2+}$ with facile synthesis: "Turn-on" response in water at neutral $\mathrm{pH}$ and its application for live cell imaging. Sens. Actuators B Chem. 2013, 184, 248-253.

[42] Lions, F.; Martin, K. V. Sexadentate Chelate Compounds. 1 X. J. Am. Chem. Soc. 1958, 80, 3858-3865.

[43] Popp, F. D. Potential anticonvulsant. XII. Anticonvulsant activity of some aldehyde derivatives. Eur. J. Med. Chem. 1989, 24, 313315.

[44] Berge, D. G. Synthesis of new 2-pyridylhydrazones and 2-quinolylhydrazones containing 2-thiophene or 2-furan groups. J. Chem. Eng. Data 1983, 28, 431-432.

[45] Sondhi, S. M.; Jain, S.; Dinodia, M.; Kumar, A. Synthesis of some thiophene, imidazole and pyridine derivatives exhibiting good anti-inflammatory and analgesic activities. Med Chem. 2008, 4, 146-154.

[46] Mosmann, T. Rapid colorimetric assay for cellular growth and survival: application to proliferation and cytotoxicity assays. J. Immunol. Methods 1983, 65, 55-63.

Received August 9, 2020 Accepted September 30, 2020 